\title{
An efficient modal control strategy for the active vibration control of a truss structure
}

\author{
Ricardo Carvalhal $^{\mathrm{a}}$, Vicente Lopes Júnior ${ }^{\mathrm{a}, *}$ and Michael J. Brennan ${ }^{\mathrm{b}}$ \\ ${ }^{a}$ Mechanical Engineering Department, Universidade Estadual Paulista, UNESP/Itha Solteira, 15385-000 Ilha \\ Solteira, SP, Brazil \\ ${ }^{\mathrm{b}}$ Institute of Sound and Vibration Research, University of Southampton, Southampton, SO17 1BJ, UK
}

Received 29 September 2005

Revised 30 August 2006

\begin{abstract}
In this paper an efficient modal control strategy is described for the active vibration control of a truss structure. In this approach, a feedback force is applied to each mode to be controlled according to a weighting factor that is determined by assessing how much each mode is excited by the primary source. The strategy is effective provided that the primary source is at a fixed position on the structure, and that the source is stationary in the statistical sense. To test the effectiveness of the control strategy it is compared with an alternative, established approach namely, Independent Modal Space Control (IMSC). Numerical simulations show that with the new strategy it is possible to significantly reduce the control effort required, with a minimal reduction in control performance.
\end{abstract}

Keywords: Intelligent truss structures, independent modal space control, piezoelectric stack actuator

\section{Introduction}

A truss structure is one of the most commonly used structures in aerospace and civil engineering [1]. Because it is desirable to use the minimum amount of material for construction, the trusses are becoming lighter and more flexible which means they are more susceptible to vibration. Passive damping is not a preferred vibration control solution because it adds weight to the system, so it is of interest to study the active control of such a structure. A convenient way of controlling a truss structure is to incorporate a piezoelectric stack actuator into one of the truss members [2]. A key issue is how many actuators to use [3], and where to place them [1]. Another issue is the dynamics of the actuators and whether these are important in terms of how they affect the vibration of the structure, and of course the control system [4,5]. Preumont et al. [6] used a local control strategy to suppress the low frequency vibrations of a truss structure using two actuators. Their strategy involved the application of integrated force feedback using two force gauges each co-located with the two piezoelectric actuators, which were fitted into different beam elements in the structure. In the work presented here, a single actuator is used and its position is determined using the method described by Carvalhal et al. [7]. The approach taken by Lammering et al. [5] is adopted to account for the actuator dynamics.

In many structures the lower modes of vibration have the most energy and are hence more critical. For this reason, these modes are often targeted for active control so that energy is not wasted in controlling the higher modes of vibration [8]. A sensible control strategy, therefore, is to model the system in terms of modal parameters and to control individual modes using modal space control strategies, such as Independent Modal Space Control (IMSC) [9]. One of the problems with this approach, however, is that large control forces can be required as identified by Singh

*Corresponding author. E-mail: vicente@ dem.feis.unesp.br. 


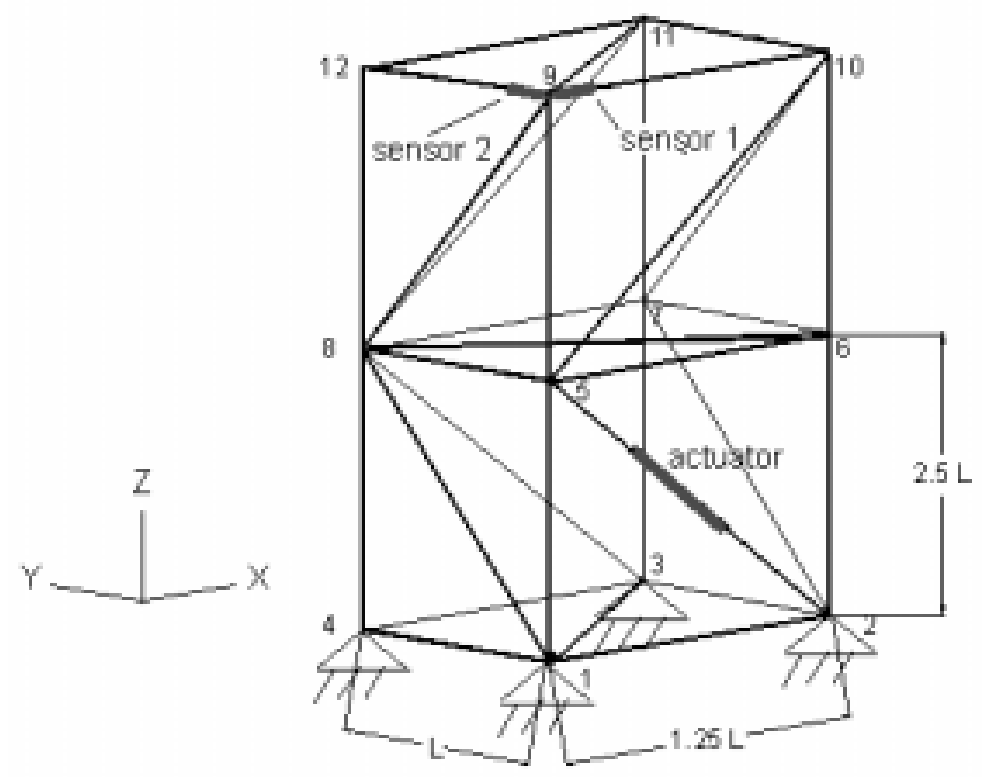

Fig. 1. Schematic of the truss structure showing the location of the actuator and sensors.

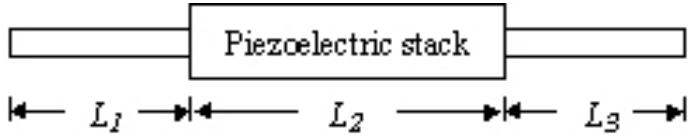

Fig. 2. Piezoelectric active member.

et al. [10]. They proposed a modification to IMSC strategy, which they called Efficient Modal Control (EMC), whereby the modal control forces are tailored according to the contribution of particular modes to the vibration at the position of interest. In the same paper, Singh et al. [10] applied the EMC technique to a cantilever beam to demonstrate the efficacy of the EMC approach.

The aim of this paper is to study the applicability of the EMC technique to control the first few modes of a truss structure. It is an extension of the work previously presented by the authors [11] and applies the control strategy suggested by Singh et al. [10] to a more realistic structure. The study involves analysis and computer simulations. First, the modal control gains are determined for the IMSC strategy. This involves the development of a state-space model of the structure from an initial finite element model, which includes both active and passive structural members. Classical Linear Quadratic Regulator (LQR) control theory is then used to determine the modal control gains. These are then modified in-line with the EMC approach, by considering the contribution of the modal amplitudes to the vibration at one point on the structure.

The paper is organized as follows. Following the introduction, Section 2 describes the way in which the statespace model of the structure is developed. In Section 3 the control strategy is outlined, and in Section 4 computer simulations demonstrate the effectiveness of the approach when applied to a truss structure. Finally, in Section 5, some general conclusions are drawn.

\section{Model of the truss structure}

In this section a state-space model of the truss structure that is suitable for control analysis is developed. The approach is similar to that described by Gawronski [12]. The structure considered consists of an active member that 
includes an actuator, and passive members, as shown in Fig. 1. A model of the passive members can be obtained using the finite element approach. With some modification it can also be used to model the active member, which consists of a piezoelectric stack actuator and two metallic bars as shown in Fig. 2. The stiffness matrix for an active member is obtained by combining the stiffness matrix for the piezoelectric actuator with the stiffness matrices of two metallic bars; each piezoelectric active member has an additional electrical degree of freedom. The mechanical-electric stiffness matrix for the piezoelectric active member, described by Lammering et al. [5], is

$$
\mathbf{K}^{\mathrm{el}}=\left[\begin{array}{ccc}
\frac{k_{1} k_{2} k_{3}}{k_{1} k_{2}+k_{1} k_{3}+k_{2} k_{3}} \frac{-k_{1} k_{2} k_{3}}{k_{1} k_{2}+k_{1} k_{3}+k_{2} k_{3}} \frac{-k_{1} c_{2} k_{3}}{k_{1} k_{2} k_{3}+k_{3} k_{3}+k_{2} k_{3}} \\
\frac{k_{1}}{k_{1} k_{2}+k_{1} k_{3}+k_{2} k_{3}} \frac{k_{1} c_{2} k_{3}}{k_{1} k_{2}+k_{1} k_{3}+k_{2} k_{3}} \frac{k_{1} c_{2} k_{3}}{k_{1} k_{2}+k_{1} k_{3}+k_{2} k_{3}} \\
\frac{-k_{1} c_{2} k_{3}}{k_{1} k_{2}+k_{1} k_{3}+k_{2} k_{3}} \frac{-c_{2}^{2}\left(k_{1}+k_{3}\right)}{k_{1} k_{2}+k_{1} k_{3}+k_{2} k_{3}} & \frac{k_{1} k_{2}+k_{1} k_{3}+k_{2} k_{3}}{k_{1}}-\bar{c}_{2}
\end{array}\right]
$$

where

$$
k_{i}=E_{i} A_{i} L_{i}, c_{2}=n_{p} e A_{2} L_{2}, \bar{c}_{2}=n_{p}^{2} \varepsilon A_{2} / L_{2}
$$

and $e$ is the piezoelectric coefficient, $\varepsilon$ is the dielectric coefficient; $E_{i}, A_{i}$ and $L_{i}$ are the elastic modulus, the cross-section area, and the length of the $i$ th part of the active member respectively; $n_{p}$ is the number of elements in the piezoelectric stack actuator. The global equations of motion for the truss structure are determined by assembling the matrices for the active and passive members to give

$$
\begin{aligned}
& \mathbf{M} \ddot{\mathbf{q}}+\mathbf{D} \dot{\mathbf{q}}+\mathbf{K} \mathbf{q}=\mathbf{B}_{\mathrm{t}} \mathbf{u}_{\mathrm{t}} \\
& \mathbf{y}=\mathbf{C}_{\mathrm{q}} \mathbf{q}+\mathbf{C}_{\dot{q}} \dot{\mathbf{q}}
\end{aligned}
$$

where $\mathbf{q}, \dot{\mathbf{q}}$ and $\ddot{\mathbf{q}}$ are $n$-length vectors of displacement, velocity and acceleration, respectively; $\mathbf{M}, \mathbf{D}$ and $\mathbf{K}$ are the $n \times n$ mass, damping, and stiffness matrices, respectively; $\mathbf{B}_{\mathrm{t}}=\left[\mathbf{B}_{\mathrm{w}} \mathbf{B}_{\mathrm{v}}\right]$ is a $n \times s$ spatial coupling matrix related to the $s$-length input vector $\mathbf{u}_{\mathrm{t}}=[\mathbf{w} \mathbf{v}]^{T}$, where $\mathbf{w}$ is the disturbance vector, $\mathbf{v}$ is the vector of applied voltages to the actuators (in the study presented here there is only one actuator), and the superscript (.) ${ }^{T}$ denotes the transpose. The $r$-length output vector is $\mathbf{y}$, which is related to the displacement and velocity vectors through the matrices $\mathbf{C}_{\mathrm{q}}$ and $\mathbf{C}_{\dot{q}}$, respectively. Assuming that the damping matrix $\mathbf{D}$ can be diagonalised, Eqs (3a) and (3b) can be written in modal coordinates as

$$
\begin{aligned}
& \ddot{\mathbf{q}}_{\mathrm{m}}+2 \mathbf{Z} \Omega \dot{\mathbf{q}}_{\mathrm{m}}+\Omega^{2} \mathbf{q}_{\mathrm{m}}=\mathbf{B}_{\mathrm{m}} \mathbf{u} \\
& \mathbf{y}=\mathbf{C}_{\mathrm{qm}} \mathbf{q}_{\mathrm{m}}+\mathbf{C}_{\dot{\mathrm{q}} \mathrm{m}} \dot{\mathbf{q}}_{\mathrm{m}}
\end{aligned}
$$

where $\mathbf{q}_{\mathrm{m}}=\Phi^{-1} \mathbf{q}$ is the vector of modal coordinates and $\Phi$ is an $n \times n$ modal matrix; $\mathbf{Z}=$ $0.5\left(\Phi^{T} \mathbf{M} \Phi\right)^{-1 / 2}\left(\Phi^{T} \mathbf{K} \Phi\right)^{-1 / 2}\left(\Phi^{T} \mathbf{D} \Phi\right)$ is the $n \times n$ damping matrix; $\Omega=\left(\Phi^{T} \mathbf{M} \Phi\right)^{-1 / 2}\left(\Phi^{T} \mathbf{K} \Phi\right)^{1 / 2}$ is the $n \times$ $n$ natural frequency matrix; $\mathbf{B}_{\mathrm{m}}=\left(\Phi^{T} \mathbf{M} \Phi\right)^{-1} \Phi^{T} \mathbf{B}_{\mathbf{t}}$ is the $n \times s$ input matrix and $\mathbf{C}_{\mathrm{qm}}=\mathbf{C}_{\mathrm{q}} \Phi, \mathbf{C}_{\dot{\mathrm{q} m}}=\mathbf{C}_{\dot{\mathrm{q}}} \Phi$ are the $r \times n$ modal displacement and velocity matrices, respectively. Equations (4a) and (4b) are a set of $n$ independent equations for each mode of the system. The equations for the $i$ th mode are given by

$$
\begin{aligned}
& \ddot{q}_{m i}+2 \zeta_{i} \omega_{i} \dot{q}_{m i}+\omega_{i}^{2} q_{m i}=\mathbf{B}_{\mathrm{mi}} \mathbf{u} \\
& \mathbf{y}_{\mathrm{i}}=\mathbf{C}_{\mathrm{qmi}} q_{m i}+\mathbf{C}_{\dot{\mathrm{q}} m i} \dot{q}_{m i}
\end{aligned}
$$

where $\mathbf{B}_{m i}$ is the $i$ th row of the modal spatial coupling matrix and $\mathbf{C}_{\mathrm{qmi}}$ and $\mathrm{C}_{\dot{\mathrm{q} m i}}$ are the $i$ th columns of the displacement and velocity matrices, respectively.

For analysis of the control system it is convenient to write the equations describing dynamics of the structure in state-space form as

$$
\dot{\mathbf{x}}=\mathbf{A x}+\mathbf{B u}
$$


Table 1

Material properties and dimensions of the smart truss structure

\begin{tabular}{lcc}
\hline & Structure & Active member \\
\hline Elastic modulus $(\mathrm{GPa})$ & 210 & 52.6 \\
Mass density $\left(\mathrm{kg} / \mathrm{m}^{3}\right)$ & 7800 & 7600 \\
Cross-section area $\left(\mathrm{m}^{2}\right)$ & $2.83 \times 10^{-5}$ & $5.65 \times 10^{-5}$ \\
$L(\mathrm{~m})$ & 0.5 & 0.25 \\
Piezoelectric coefficient (N/V.m) & - & 44 \\
Dielectric coefficient $(\mathrm{F} / \mathrm{m})$ & - & $2.12 \times 10^{-8}$ \\
\hline
\end{tabular}

Table 2

Optimal feedback gains for the controlled modes

\begin{tabular}{ccc}
\hline Mode number $(i)$ & $G_{d i}\left(10^{3}\right)$ & $G_{v i}\left(10^{3}\right)$ \\
\hline 1 & 1.00 & 0.44 \\
2 & 5.02 & 0.45 \\
3 & 2.98 & 0.43 \\
4 & -1.05 & -0.38 \\
\hline
\end{tabular}

$$
\mathbf{y}=\mathbf{C x}
$$

where $\mathbf{A}$ is the $2 n \times 2 n$ dynamic matrix, $\mathbf{B}$ is the $2 n \times s$ input matrix and $\mathbf{C}$ is the $r \times 2 n$ output matrix. The state vector $\mathbf{x}$ of modal displacements and velocities consists of $2 n$ independent components, $\mathbf{x}_{i}$, that represent the state of each mode and are given by $\mathbf{x}_{\mathrm{i}}=\left\{\begin{array}{c}q_{m i} \\ \dot{q}_{m i}\end{array}\right\}$, where $q_{m i}$ is the $i$ th modal displacement and $\dot{q}_{m i}$ is the $i$ th modal velocity. The modal state-space realization is characterized by the block-diagonal dynamic matrix and the related input and output matrices given by

$$
\mathbf{A}=\operatorname{blockdiag}\left(\mathbf{A}_{\mathrm{mi}}\right), \mathbf{B}=\left[\begin{array}{l}
\vdots \\
\mathbf{B}_{\mathrm{mi}} \\
\vdots
\end{array}\right], \mathbf{C}=\left[\begin{array}{l}
\left.\mathbf{C}_{\mathrm{mi}} \cdots\right] \\
\cdots
\end{array}\right]
$$

where $\mathbf{A}_{\mathrm{mi}}=\left[\begin{array}{ll}0 & 1 \\ -\omega_{i}^{2}-2 \zeta_{i} \omega_{i}\end{array}\right]$, and $\mathbf{B}_{\mathrm{mi}}$ and $\mathbf{C}_{\mathrm{mi}}$ are $2 \times s$, and $r \times 2$ blocks respectively, $\zeta_{i}$ is the $i$ th modal damping ratio, $\omega_{i}$ is the $i$ th natural frequency, and the subscript (.) mi relates to the $i$ th mode.

The order of the state-space modal is large. However, a low order model is essential for the successful implementation of a controller. This can be obtained by partitioning the modes into those that will be controlled and those that will not be controlled. Thus, Eqs (6a) and (6b) can be written as.

$$
\begin{aligned}
& \left\{\begin{array}{l}
\dot{x}_{\mathbf{c}} \\
\dot{x}_{\mathbf{r}}
\end{array}\right\}=\left[\begin{array}{ll}
\mathrm{A}_{\mathbf{c}} & 0 \\
\mathbf{0} & \mathrm{A}_{\mathbf{r}}
\end{array}\right]\left\{\begin{array}{l}
\mathrm{x}_{\mathbf{c}} \\
\mathrm{x}_{\mathbf{r}}
\end{array}\right\}+\left[\begin{array}{l}
\mathrm{B}_{\mathbf{c}} \\
\mathrm{B}_{\mathbf{r}}
\end{array}\right] \mathbf{u} \\
& \mathbf{y}=\left[\mathbf{C}_{\mathbf{c}} \mathbf{C}_{\mathbf{r}}\right]\left\{\begin{array}{l}
\mathrm{x}_{\mathbf{c}} \\
\mathrm{x}_{\mathbf{r}}
\end{array}\right\}
\end{aligned}
$$

where the subscripts $(.)_{\mathrm{c}}$ and $(.)_{\mathrm{r}}$ are for the controlled and residual modes, respectively.

\section{Efficient modal control (EMC)}

This control strategy is a simple modification of the IMSC, developed by Meirovitch and Baruh [9], and a description of it is given in this section. As mentioned in the introduction, this method has been applied to a cantilever beam [10], and here it is applied to a truss structure.

In IMSC the control force vector in physical space is given by (Jia [13])

$$
\mathbf{u}_{\mathrm{t}}=\mathbf{L}_{\mathrm{c}} \mathbf{u}, \text { where } \mathbf{L}_{\mathrm{c}}=\left(\mathbf{L}^{T} \mathbf{L}\right)^{-1} \mathbf{L}^{T}
$$




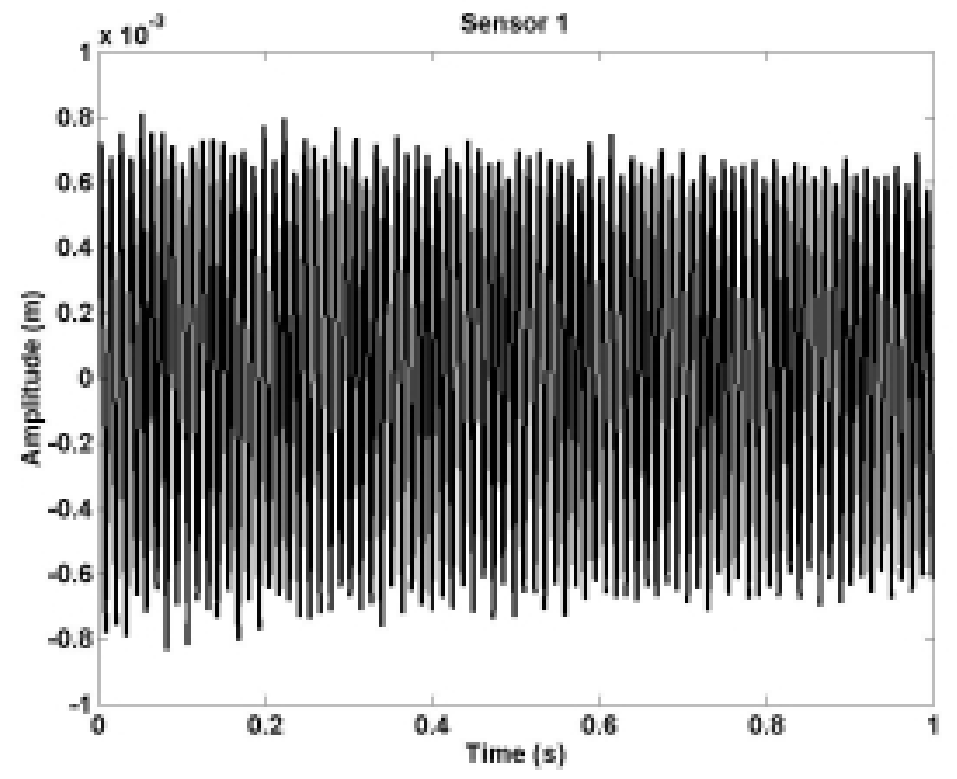

(a)

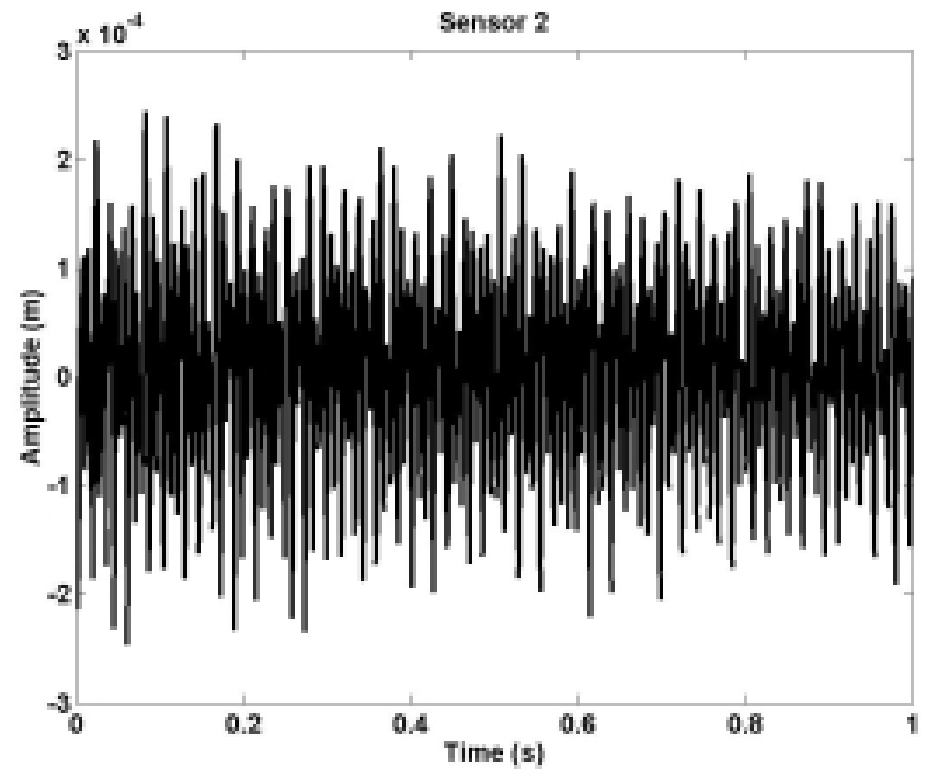

(b)

Fig. 3. Uncontrolled response for the reduced model with an impulsive force at (a) sensor 1 and (b) sensor 2.

where $\mathbf{L}=\left(\Phi^{T} \mathbf{M} \Phi\right)^{-1} \Phi^{T} \mathbf{B}_{\mathrm{t}}$. An independent controller can be designed for each mode, hence the modal feedback force in each mode $u_{i}$ is dependent on $q_{m i}$ and $\dot{q}_{m i}$ alone, so that

$$
u_{i}=-G_{d i} q_{m i}-G_{v i} \dot{q}_{m i}
$$

where $G_{d i}$ and $G_{v i}$ are the displacement and velocity modal gains, respectively. This ensures that the modal equations 


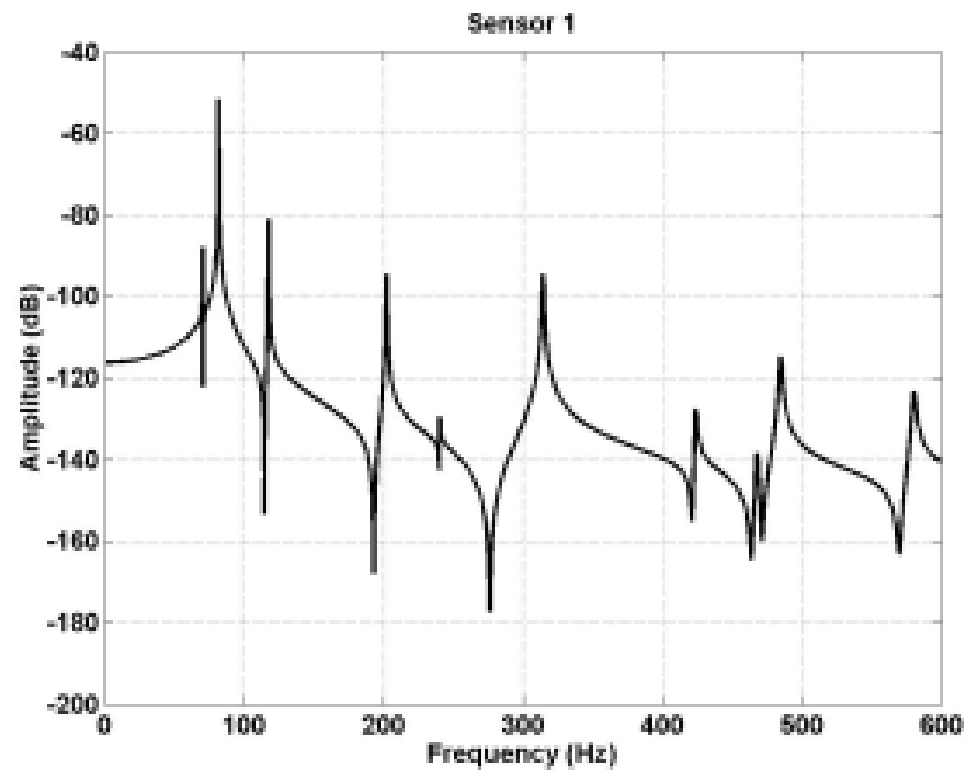

(a)

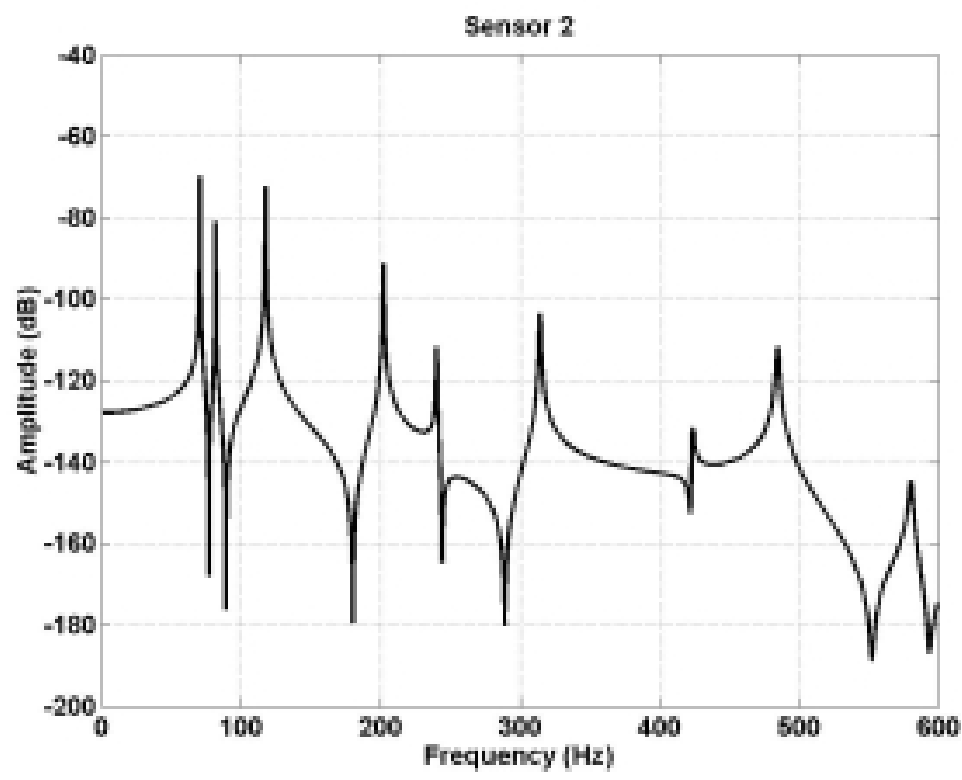

(b)

Fig. 4. Spectra of the uncontrolled response for (a) sensor 1 and (b) sensor 2.

are not coupled through feedback. The modal control forces $u_{i}$ are determined using optimal control theory [14], which determines feedback control gains by minimizing a quadratic performance index $J_{i}$ given by

$$
J_{i}=\frac{1}{2} \int_{0}^{\infty}\left(\mathbf{x}_{\mathrm{i}}^{T} \mathbf{Q}_{\mathrm{i}} \mathbf{x}_{\mathrm{i}}+R_{i} u_{i}^{2}\right) d t=\frac{1}{2} \int_{0}^{\infty}\left(\omega_{i}^{2} q_{m i}^{2}+\dot{q}_{m i}^{2}+R_{i} u_{i}^{2}\right) d t
$$




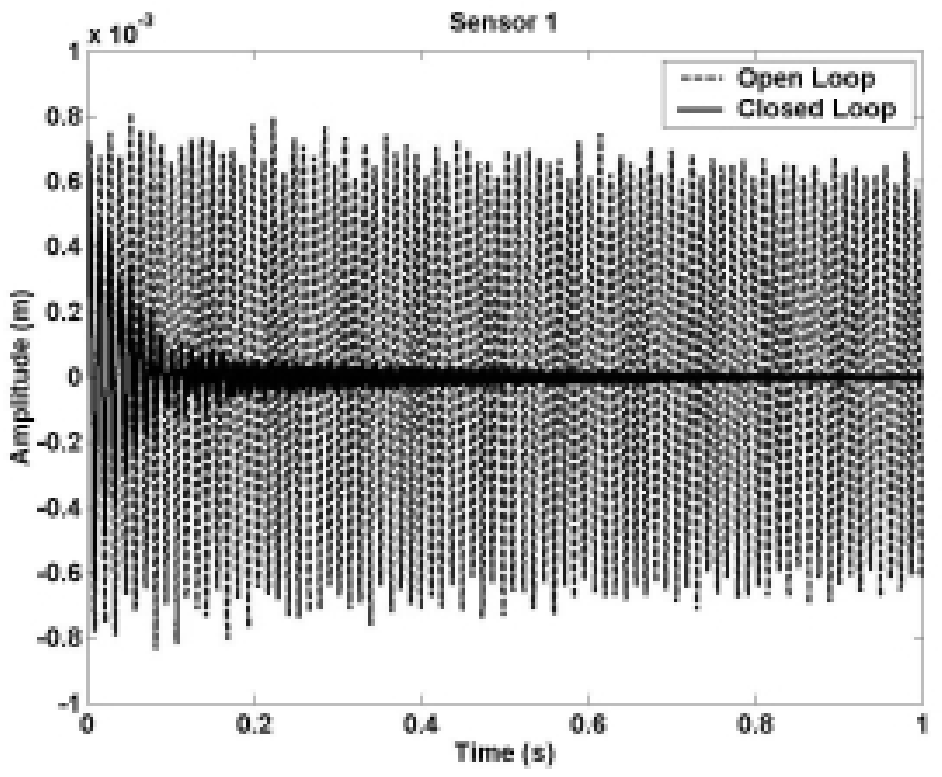

(a)

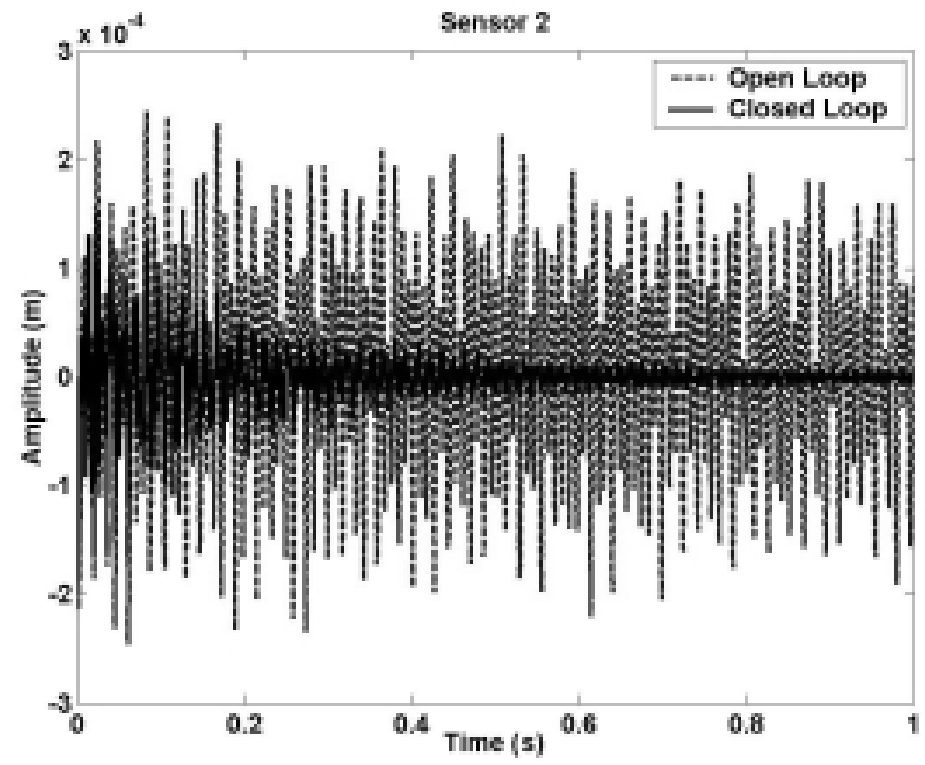

(b)

Fig. 5. Uncontrolled and controlled response for the structure at (a) sensor 1 and (b) sensor 2, when the IMSC strategy is implemented.

where the diagonal matrix $\mathbf{Q}_{i}$ is the weighting matrix for the $i$ th modal state vector; $R_{i}$ is the weighting factor for the $i$ th modal control force, which trades control performance with control effort. When $\mathbf{Q}_{\mathrm{i}}=\left[\begin{array}{cc}\omega_{i}^{2} & 0 \\ 0 & 1\end{array}\right]$, the performance index is related to the sum of the potential energy $\omega_{i}^{2} q_{m i}^{2}$ and the kinetic energy $\dot{q}_{m i}^{2}$ of the vibrating system as well as the required input control effort $u_{i}^{2}$. The $i$ th modal control force can be determined by 
Table 3

Performance of the IMSC and EMC approaches for the ten first modes

\begin{tabular}{|c|c|c|c|c|c|c|c|c|c|c|c|}
\hline \multirow{2}{*}{ Modes } & \multicolumn{3}{|c|}{ Uncontrolled } & \multicolumn{4}{|c|}{ IMSC } & \multicolumn{4}{|c|}{ EMC } \\
\hline & $\begin{array}{c}\text { Frequency } \\
(\mathrm{Hz})\end{array}$ & $\begin{array}{l}\text { Mag. } \\
(\mathrm{dB})\end{array}$ & $\begin{array}{c}\text { Damping } \\
\text { ratio }\left(10^{-4}\right)\end{array}$ & $\begin{array}{c}\text { Frequency } \\
(\mathrm{Hz})\end{array}$ & $\begin{array}{l}\text { Mag. } \\
(\mathrm{dB})\end{array}$ & $\begin{array}{c}\text { Damping } \\
\text { ratio }\left(10^{-4}\right)\end{array}$ & $\begin{array}{l}\text { Attenuation } \\
\text { (dB) }\end{array}$ & $\begin{array}{l}\text { Frequency } \\
(\mathrm{Hz})\end{array}$ & $\begin{array}{l}\text { Mag. } \\
(\mathrm{dB})\end{array}$ & $\begin{array}{c}\text { Damping } \\
\text { ratio }\left(10^{-4}\right)\end{array}$ & $\begin{array}{l}\text { Attenuation } \\
\text { (dB) }\end{array}$ \\
\hline 1 & 70.5 & -71.6 & 2.2 & 70.8 & -102.5 & 91.7 & -30.9 & 70.6 & -103.0 & 99.8 & -31.4 \\
\hline 2 & 81.8 & -81.2 & 2.6 & 81.8 & -125.9 & 346.0 & -44.7 & 81.8 & -117.0 & 134.0 & -35.8 \\
\hline 3 & 117.6 & -72.4 & 3.7 & 117.3 & -107.2 & 178.6 & -34.8 & 117.6 & -104.8 & 144.7 & -32.4 \\
\hline 4 & 202.1 & -92.2 & 6.3 & 202.1 & -106.5 & 37.2 & -14.3 & 202.1 & -99.2 & 15.7 & -7.0 \\
\hline 5 & 240.3 & -112.9 & 7.6 & 240.3 & -107.3 & 4.3 & +5.6 & 240.3 & -111.5 & 6.8 & +1.4 \\
\hline 6 & 315.1 & -103.9 & 9.9 & 315.1 & -104.8 & 10.4 & -0.9 & 315.1 & -104.5 & 10.1 & -0.6 \\
\hline 7 & 423.4 & -133.1 & 13.3 & 423.4 & -130.2 & 13.4 & +2.9 & 423.4 & -131.8 & 13.3 & +1.3 \\
\hline 8 & 467.9 & -136.3 & 14.7 & 467.9 & -136.3 & 14.7 & 0.0 & 467.9 & -136.3 & 14.7 & 0.0 \\
\hline 9 & 483.8 & -112.0 & 15.2 & 483.8 & -112.0 & 15.2 & 0.0 & 483.8 & -112.0 & 15.2 & 0.0 \\
\hline 10 & 580.9 & -145.3 & 18.2 & 580.9 & -145.3 & 18.2 & 0.0 & 580.9 & -145.3 & 18.2 & 0.0 \\
\hline
\end{tabular}

$$
u_{i}=-R_{i}^{-1} \mathbf{B}_{\mathrm{mi}}^{T} \mathbf{P}_{\mathrm{i}} \mathbf{x}_{\mathrm{i}}
$$

where $\mathbf{B}_{\mathrm{mi}}^{T}$ is the part of the matrix $\mathbf{B}$ in Eq. (4a) that is related to the $i$ th control input, $\mathbf{P}_{\mathbf{i}}$ is a semi-definite symmetric matrix, and it is governed by the Riccati equation (Xu and Jiang [15])

$$
\mathbf{P}_{\mathrm{i}} \mathbf{A}_{\mathrm{mi}}+\mathbf{A}_{\mathrm{mi}}^{T} \mathbf{P}_{\mathrm{i}}+\mathbf{Q}_{\mathrm{i}}-\mathbf{P}_{\mathrm{i}} \mathbf{B}_{\mathrm{mi}} R_{i}^{-1} \mathbf{B}_{\mathrm{mi}}^{-1} \mathbf{P}_{\mathrm{i}}=0
$$

Since all modal states are not available for feedback in Eq. (12), the estimated modal states $\hat{\mathbf{x}}_{\mathrm{i}}$ are used instead and the modal control force becomes

$$
\mathbf{u}=-\mathbf{G} \hat{\mathbf{x}}_{\mathrm{c}}
$$

where $\mathbf{G}$ is the controller gain matrix. To estimate the full controlled modal state $\hat{\mathbf{x}}_{\mathrm{c}}$ from the output $\mathbf{y}$, a deterministic modal observer can be used [14], which is described by

$$
\hat{\mathbf{x}}_{\mathrm{c}}=\mathbf{A}_{\mathrm{c}} \hat{\mathbf{x}}_{\mathrm{c}}+\mathbf{B}_{\mathrm{c}} \mathbf{u}_{\mathrm{c}}+\mathbf{H}\left[\mathbf{y}-\mathbf{C}_{\mathrm{c}} \hat{\mathbf{x}}_{\mathrm{c}}\right]
$$

where $\mathbf{H}$ is the observer gain matrix, which can be determined optimally by solving a matrix Riccati equation. For a time-invariant modal observer, the Riccati matrix satisfies the algebraic matrix Riccati equation

$$
\mathbf{S A}_{\mathrm{c}}^{T}+\mathbf{A}_{\mathrm{c}} \mathbf{S}-\mathbf{S C}_{\mathrm{c}}^{T} \mathbf{W}^{-1} \mathbf{C}_{\mathrm{c}} \mathbf{S}+\mathbf{V}=0
$$

where $\mathbf{S}$ is the Riccati equation solution. Equation (16) yields the optimal observer gain matrix $\mathbf{H}$ for the $\mathbf{V}$ and $\mathbf{W}$ weighting matrices

$$
\mathbf{H}=\mathbf{S C}_{\mathrm{c}}^{T} \mathbf{W}^{-1}
$$

The error vector, $\mathbf{e}_{\mathrm{c}}=\hat{\mathbf{x}}_{\mathrm{c}}-\mathbf{x}$, can be written as

$$
\dot{\mathbf{e}}_{\mathrm{c}}=\left(\mathbf{A}_{\mathrm{c}}-\mathbf{H C}_{\mathrm{c}}\right) \mathbf{e}_{\mathrm{c}}+\mathbf{H C}_{\mathrm{r}} \mathbf{x}_{\mathrm{r}}
$$

Combining Eqs (8a), (14) and (18) the system of equations can be written in matrix form as

$$
\left\{\begin{array}{c}
\dot{\mathbf{x}}_{\mathrm{c}} \\
\dot{\mathbf{x}}_{\mathrm{r}} \\
\dot{\mathbf{e}}_{\mathrm{c}}
\end{array}\right\}=\left[\begin{array}{ccc}
\mathbf{A}_{\mathrm{c}}-\mathbf{B}_{\mathrm{c}} \mathbf{G} & \mathbf{0} & -\mathbf{B}_{\mathrm{c}} \mathbf{G} \\
-\mathbf{B}_{\mathrm{r}} \mathbf{G} & \mathbf{A}_{\mathrm{r}} & -\mathbf{B}_{\mathrm{r}} \mathbf{G} \\
\mathbf{0} & -\mathbf{H} \mathbf{C}_{\mathrm{r}} & \mathbf{A}_{\mathbf{c}}-\mathbf{H C}_{\mathrm{c}}
\end{array}\right]\left\{\begin{array}{c}
\mathbf{x}_{\mathrm{c}} \\
\mathbf{x}_{\mathrm{r}} \\
\mathbf{e}_{\mathrm{c}}
\end{array}\right\}
$$

The term $-\mathbf{B}_{\mathrm{r}} \mathbf{G}$ is the cause of the residual modes being excited by the control forces, which is known as control spillover. This term has no effect on the eigenvalues of the closed-loop system and it cannot destabilize the system, although it can cause some degradation in the performance. On the other hand, the term $-\mathbf{H C} \mathbf{C}_{\mathrm{r}}$ can cause instability in the residual modes, which is known as observation spillover. However, a small amount of modal damping, inherent in the structure can help to reduce the effects of observation spillover. Another way to minimize this effect is to use a large number of sensors or to filter the sensor signals, in order to diminish the contribution of residual modes [14].

Equation (19) can be solved to determine the closed-loop response of the system in any mode. Fang et al. [8] show in a recent study that the modal control forces may increase the amplitudes of higher modes of vibration (uncontrolled 


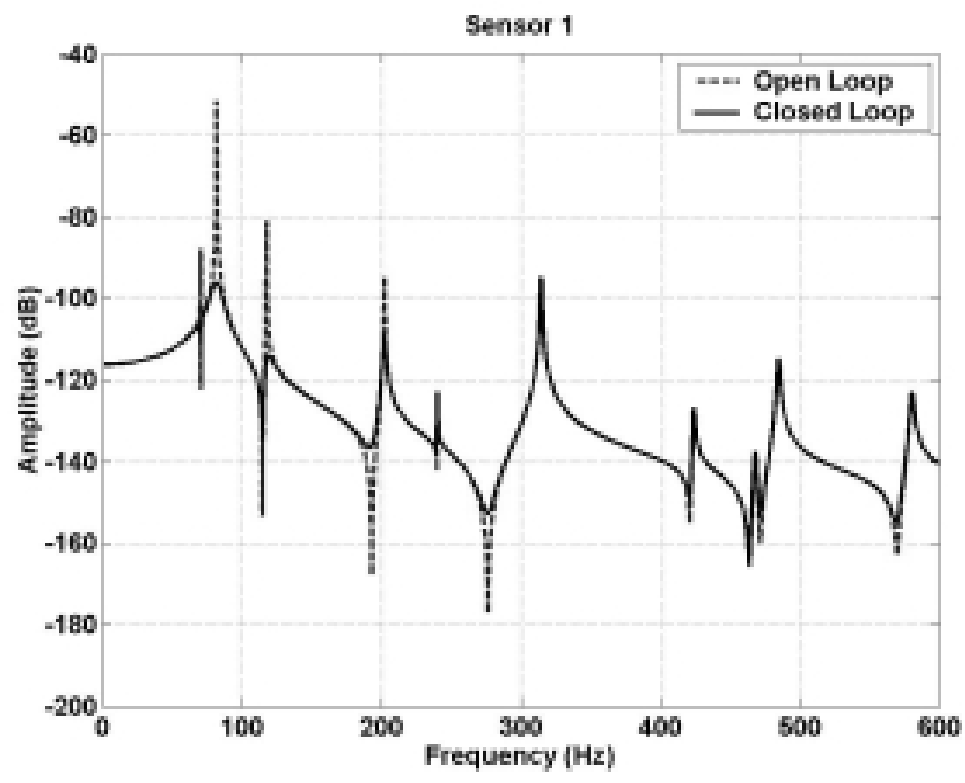

(a)

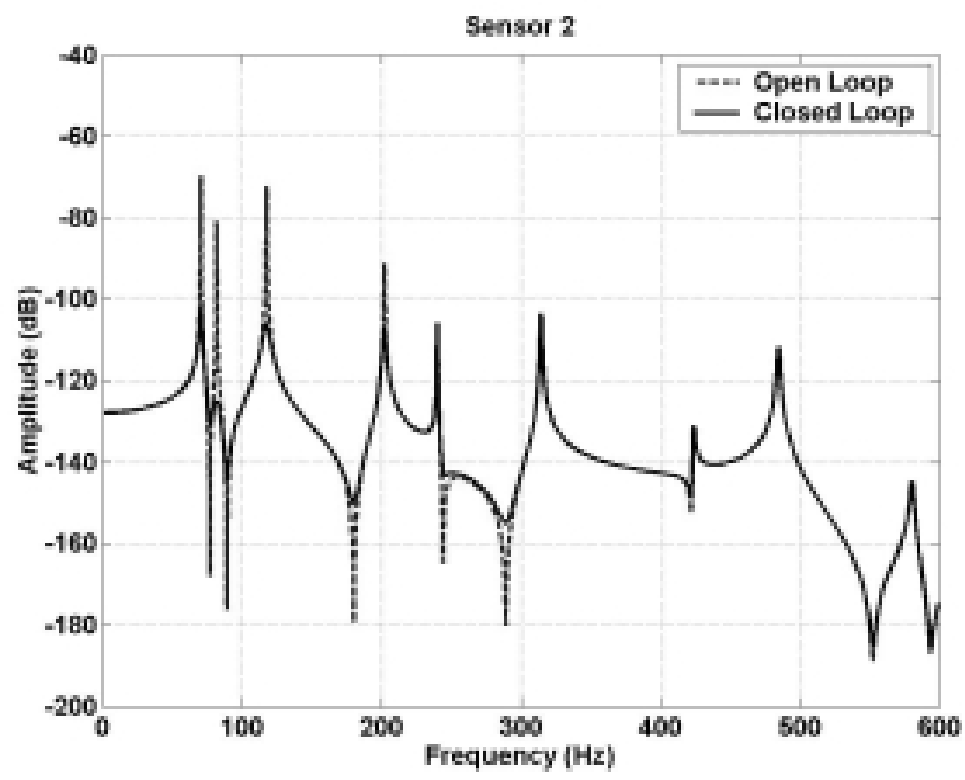

(b)

Fig. 6. Spectra of the uncontrolled and controlled system at (a) sensor 1 and (b) sensor 2 when the IMSC strategy is implemented.

modes) if the IMSC algorithm is used to design a control system for a multi-degree-of-freedom structural system. Thus, the effects of control forces on higher modes must be considered in the response analysis.

The analysis above relates to IMSC. As mentioned previously, one of the problems with this control strategy is that if a number of modes are excited, the voltages supplied to the control actuator(s) can be very large [10]. A significant reduction in the control force can be achieved with only a small reduction in performance, if the primary excitation 


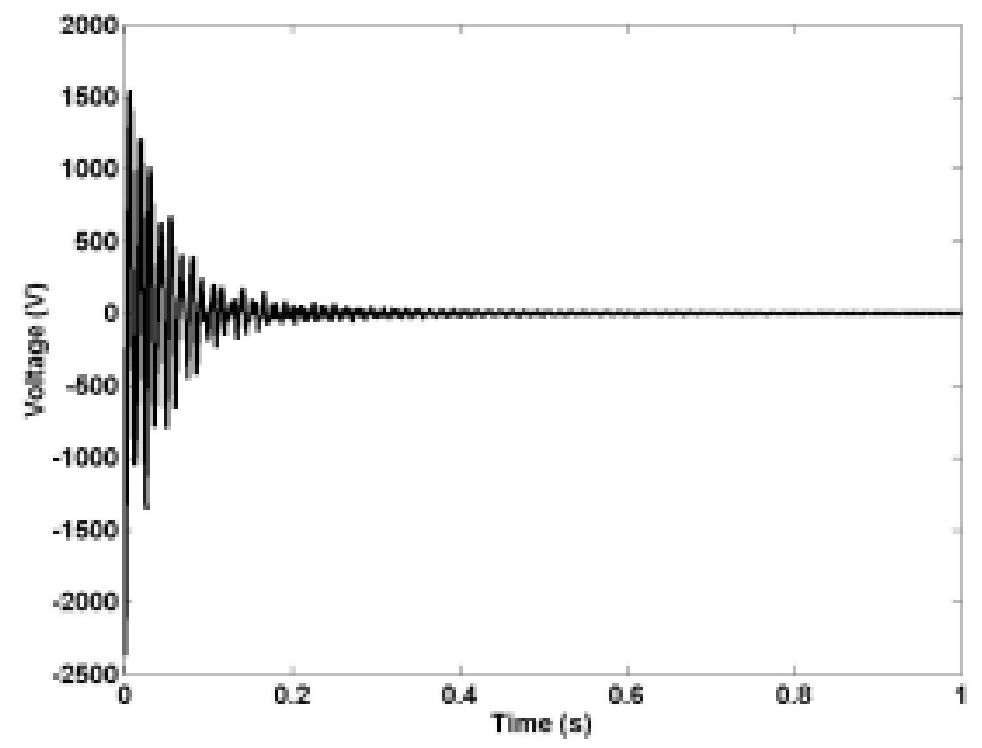

Fig. 7. Feedback control voltage applied by the piezoelectric actuator when the IMSC strategy is implemented.

is at a fixed position on the structure, and if this source is stationary in the statistical sense. The information about the uncontrolled system response can be utilized to tailor the control forces. Singh et al. [10] observed that when the IMSC strategy was implemented on a cantilever beam, the optimal feedback gains were higher for higher modes of vibration, and the amplitude of vibration was generally lower in these modes. In the truss structure, the increase in control force amplitude with frequency for IMSC was not observed. This is probably because the modal gains are dependent on the type of structure and the placement of the actuators and sensors.

The EMC control strategy simply weights the control gains for each mode according to their contribution to the overall vibration level at a particular location on the structure. The ratios of the amplitudes of different modes are first calculated with respect to the mode having maximum amplitude, when the structure is excited by the primary excitation source alone. The feedback gains are then weighted such that the modes that have smaller amplitudes have a smaller gain. Thus the gains for the $i$ th, $j$ th and $k$ th modes are determined by

$$
G_{r}(i): G_{r}(j): G_{r}(k)=1: \frac{\operatorname{displacement}(j)}{\operatorname{displacement}(i)}: \frac{\operatorname{displacement}(k)}{\operatorname{displacement}(i)}
$$

where $G_{r}(i)$ is the gain ratio of the mode $i$, and the $i$ th mode has the maximum amplitude.

\section{Numerical simulations}

Numerical simulations are presented to demonstrate the efficacy of the EMC approach applied to a truss structure, and the results are compared to the IMSC approach on the same structure. The structure considered is a 2-bay truss structure with 31 members as shown in Fig. 1; nodes 1 to 4 are clamped. The passive members are made of steel with a diameter of $6 \mathrm{~mm}$, and the damping is assumed to be proportional to the stiffness and mass matrices so that $\mathbf{D}=10^{-6} . \mathbf{K}+10^{-4}$. M. At each node there is a centralized mass block of $0.3 \mathrm{~kg}$, and the piezoelectric actuator comprises 250 circular piezoelectric elements. The material properties and dimensions are given in Table 1. Each node has three degrees of freedom (dof), translation in $x, y$ and $z$ directions, so the truss structure has 24 active dofs, and the state-space model consequently has an order of 48 . The strategy is to control the first four modes (8 states), with the sixteen remaining modes (32 states) being considered as residual modes.

The optimal positioning of the active member with the objective of controlling the first four modes of this structure, using one actuator and four sensors (to estimate the modal states), was developed using the $\mathrm{H}_{\infty}$ norm for each 


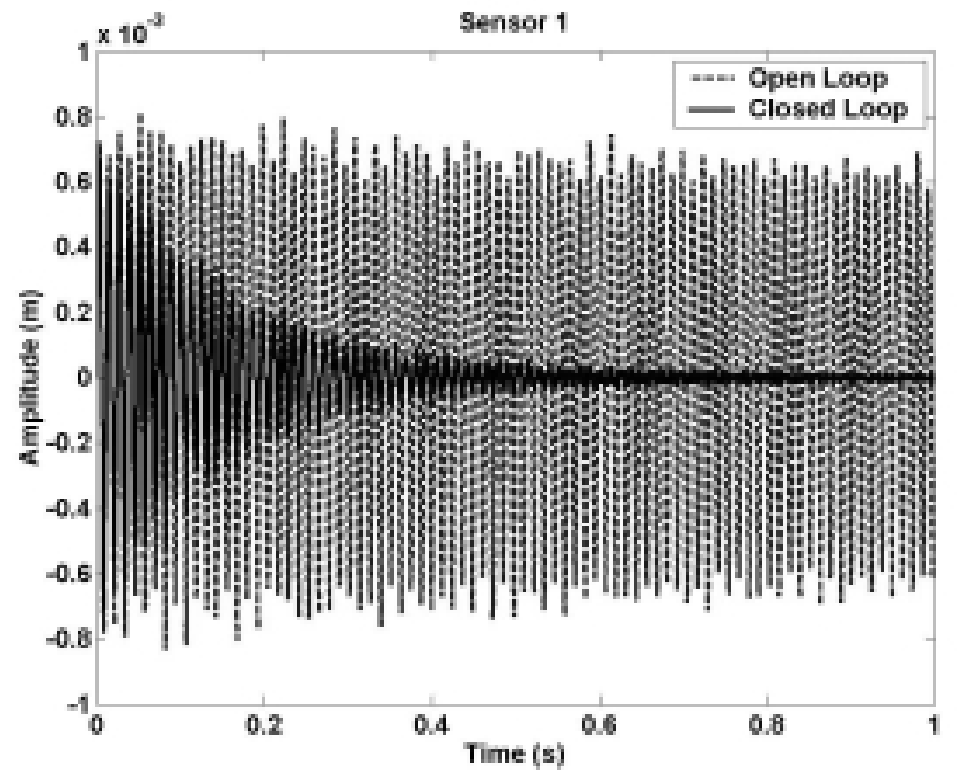

(a)

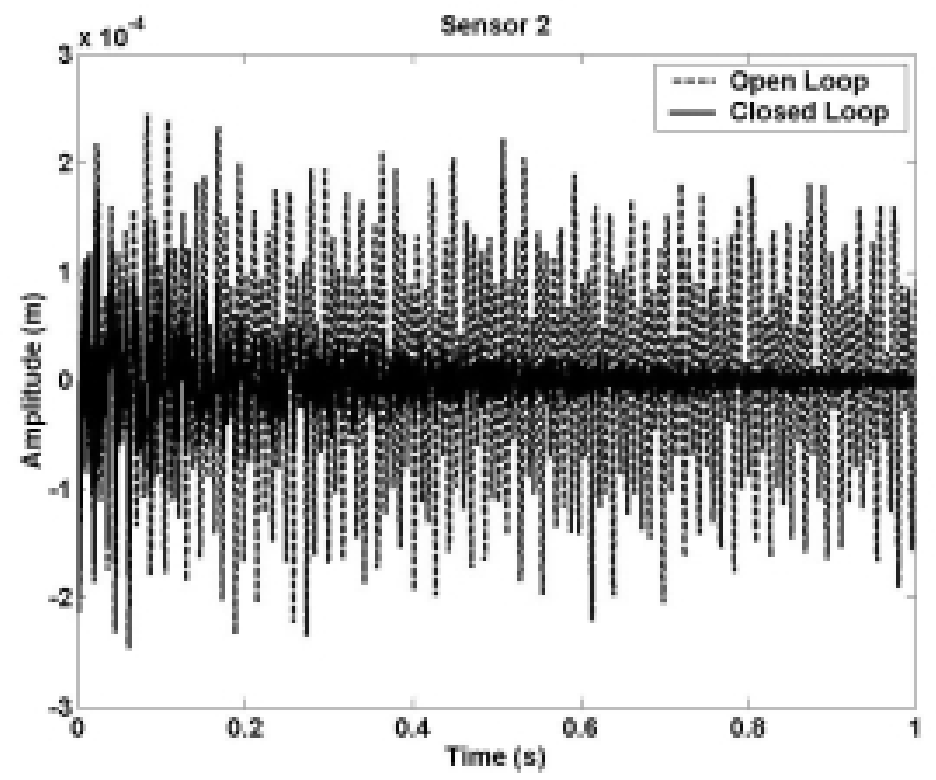

(b)

Fig. 8. Uncontrolled and controlled response for the structure at (a) sensor 1 and (b) sensor 2, when the EMC strategy is implemented.

sensor/actuator candidate position. This methodology is described by Carvalhal et al. [7]. These positions correspond to a situation where the actuator and sensors couple into and sense the motion to be controlled effectively. The optimal placement of the actuator and of the two sensors (to show the control performance) is shown in Fig. 1. The four sensors used for the observer design are located at nodes 5 and 9 in the $x$ and $y$ directions.

An impulsive force is applied at node 9 in the $x$ direction of the structure, which excites some modes of the system. 


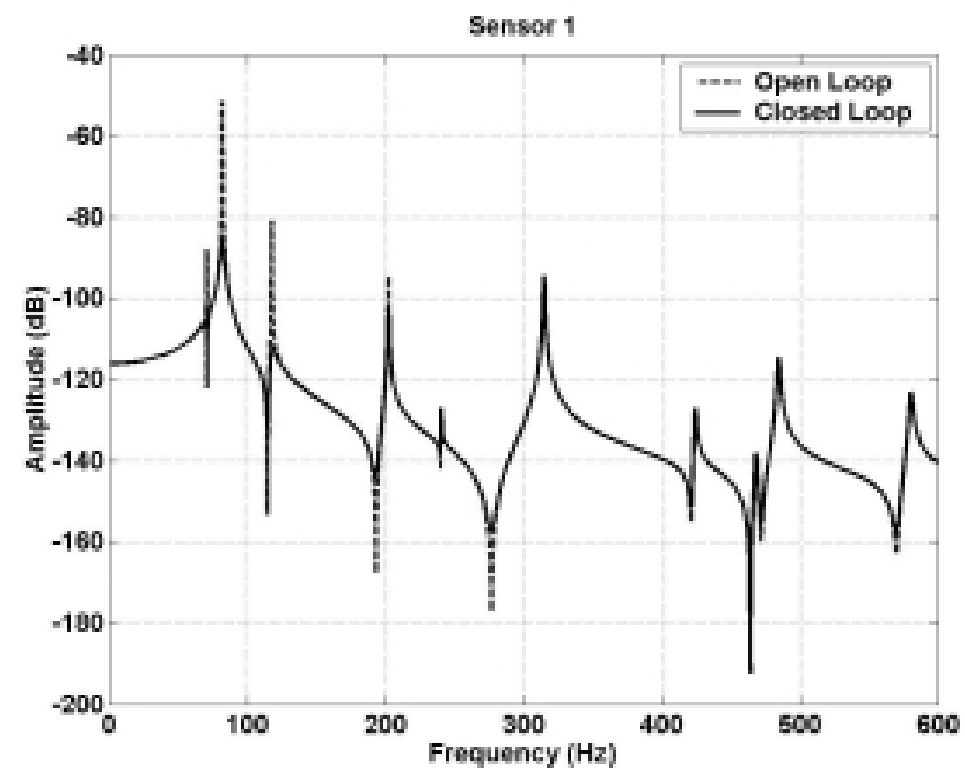

(a)

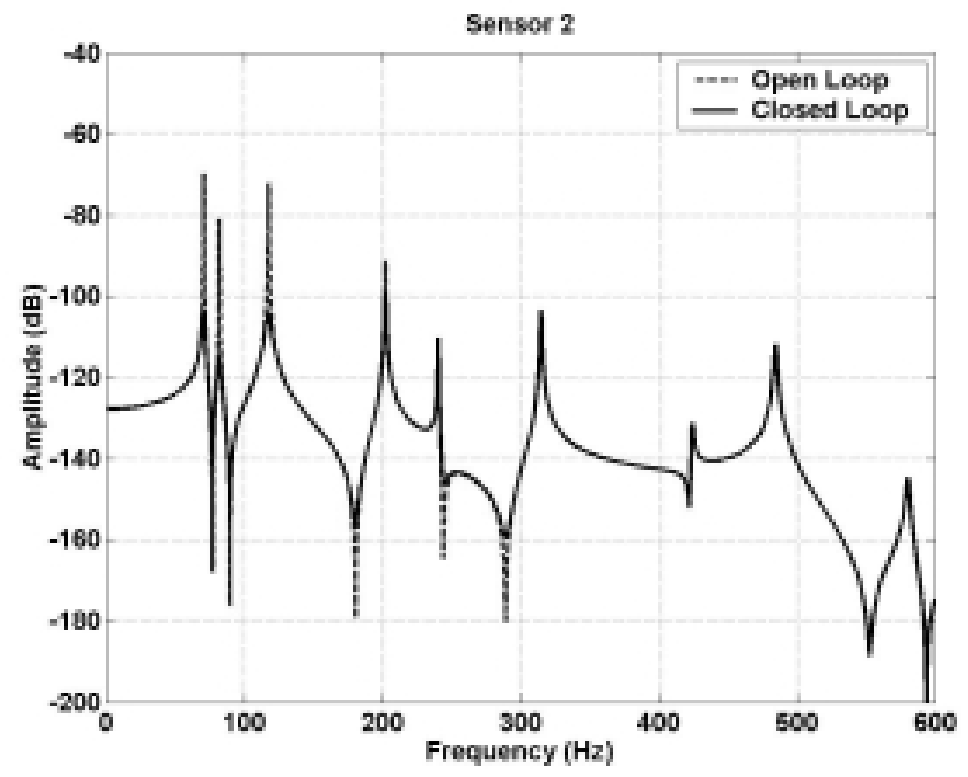

(b)

Fig. 9. Spectra of the uncontrolled and controlled system at (a) sensor 1 and (b) sensor 2 when the EMC strategy is implemented.

This type of force is used as it will excite many modes of vibration and hence will be a difficult test for the control system. The uncontrolled responses of the truss structure in the time domain at sensors 1 and 2 are shown in Fig. 3. The spectra of the uncontrolled responses are shown in Fig. 4. It should be noted that the response at sensor 1 is much larger than that at sensor 2. Using Eq. (12) with $R=10^{-5}$ IMSC feedback gains for the four controlled modes of the structure can be calculated and are given in Table 2. The actuator forces for different modes are summed to 


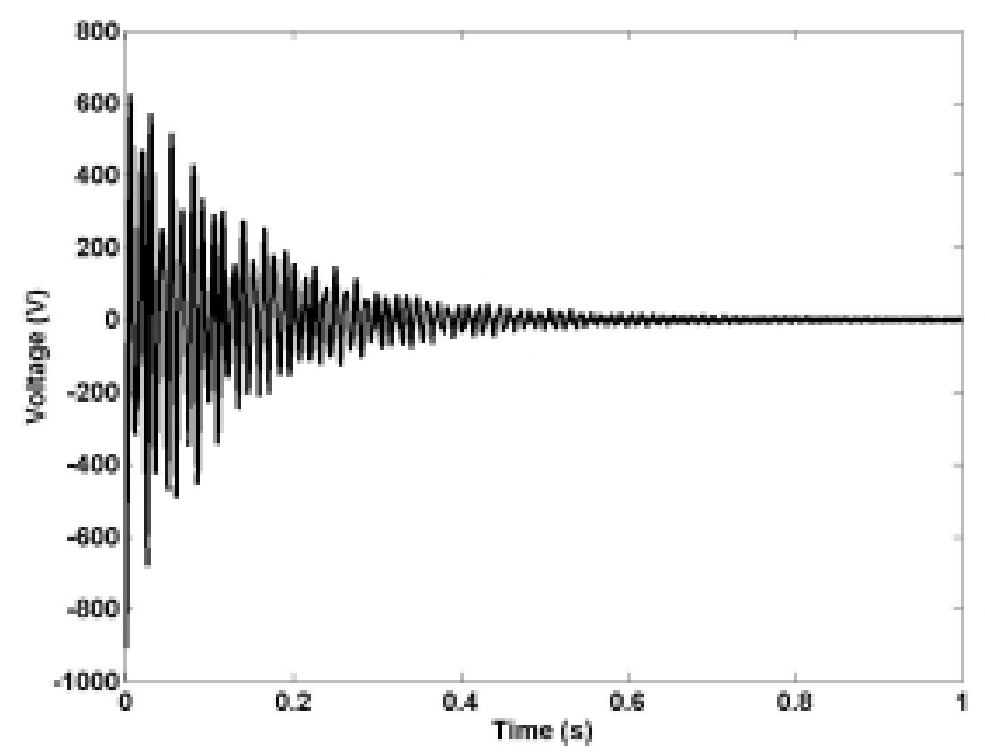

Fig. 10. Feedback control voltage applied by the piezoelectric actuator when the EMC strategy is implemented.

give the resultant force required and hence the total voltage applied can be calculated. Figure 5 shows the controlled response of the truss structure measured by both sensors. With this configuration and weighting factor, the overall amplitude decreases from the initial value of about $0.8 \times 10^{-3}(\mathrm{~m})$ to $0.5 \times 10^{-4}(\mathrm{~m})$ in about $0.3 \mathrm{~s}$ measured by sensor 1 and from $2.5 \times 10^{-4}(\mathrm{~m})$ to $0.4 \times 10^{-4}(\mathrm{~m})$ in about $0.3 \mathrm{~s}$ measured by sensor 2 . Figure 6 compares the spectra of uncontrolled and controlled responses at sensors 1 and 2. Figure 7 shows the feedback voltage supplied to the actuator. The maximum control voltage required is $2350 \mathrm{~V}$.

From the response spectra at sensor 2 shown in Fig. 4(b), the displacement amplitudes in the first four modes are found to be $[32.2,9.1,24.5,2.8] \times 10^{-5} \mathrm{~m} / \mathrm{N}$, respectively. Thus, optimal gains in these modes for the EMC control strategy were reduced by the ratios given in Eq. (20). Figure 8 shows the response of the structure when these gains are applied. For EMC, the amplitude at sensor 1 decreases from the initial value of about $0.8 \times 10^{-3}(\mathrm{~m})$ to $1.2 \times$ $10^{-4}(\mathrm{~m})$ in about $0.3 \mathrm{~s}$ and from $2.5 \times 10^{-4}(\mathrm{~m})$ to $0.6 \times 10^{-4}(\mathrm{~m})$ in about $0.3 \mathrm{~s}$ at sensor 2 . Figure 9 compares the spectra of the uncontrolled and controlled system at sensors 1 and 2 . The maximum control voltage supplied to the actuator with the EMC approach is $900 \mathrm{~V}$, and is shown in Fig. 10.

Table 3 lists the vibration attenuation in the first ten modes for the IMSC and EMC approaches, compared with the uncontrolled system. It can be seen that in both approaches the control systems are effective. The amplitude of the first mode is attenuated by about $31 \mathrm{~dB}$ in each case. With the EMC strategy, the second, third and fourth modes are attenuated less than for the IMSC strategy as expected, but the performance is still very good. Furthermore, the controller does not influence the amplitude of mode 6, which is not explicitly included in the controller. Some peaks increase, however, for example the amplitudes of modes 5 and 7 increase by $5.6 \mathrm{~dB}$ and $2.9 \mathrm{~dB}$, respectively, for the IMSC strategy, and by $1.4 \mathrm{~dB}$ and $1.3 \mathrm{~dB}$ respectively for the EMC strategy. This occurs, because of control spillover.

A single quantity can be used as an overall judge of the control performance. It is the area under the curve of the absolute value of the displacement response. For the responses in Fig. 3 this is $17.5 \times 10^{-5} \mathrm{~m} . \mathrm{s}$ and $3.75 \times$ $10^{-5} \mathrm{~m} . \mathrm{s}$ for sensor 1 and sensor 2, respectively. For the IMSC strategy (Fig. 5), these values are $3.10^{\circ} \times 10^{-5} \mathrm{~m} . \mathrm{s}$ for sensor 1 and $1.28 \times 10^{-5} \mathrm{~m} . \mathrm{s}$ for sensor 2, giving a reduction of $82.3 \%$ and $65.9 \%$, respectively. For the EMC strategy (Fig. 8), the values are $7.15 \times 10^{-5} \mathrm{~m} . \mathrm{s}$ for sensor 1 and $1.91 \times 10^{-5} \mathrm{~m} . \mathrm{s}$ for sensor 2, giving a reduction of $59.1 \%$ and $49.1 \%$, respectively. Figures 5 and 8 shows that EMC and IMSC have comparable settling times, $0.58 \mathrm{~s}$ for EMC and $0.44 \mathrm{~s}$ for IMSC. The difference lies in the vibration attenuation of the second, third and fourth modal amplitudes, for which there is much reduced control effort with the EMC approach. Figures 7 and 10 clearly 
show that the maximum feedback voltage supplied to the actuator for the EMC strategy is reduced by a factor of 2.6 compared with the IMSC strategy. Also, the voltage amplitudes at the settling times are $35.7 \mathrm{~V}$ and $21.4 \mathrm{~V}$ for the IMSC and EMC strategy respectively, i.e., it is reduced by a factor of 1.6.

\section{Conclusions}

In this paper, an efficient modal control strategy has been applied to a truss structure, and its effectiveness has been compared to that of the IMSC strategy. Optimal modal feedback gains are independent of the applied force for the IMSC algorithm, but by tailoring the gains according to the modal amplitudes at the location of interest, which are dependent on the applied force, significant reductions in the control force can be achieved, without a commensurate loss in performance. Simulations on a truss structure have demonstrated that, provided the nature of the primary excitation is known and is stationary, the simple modification of the IMSC strategy is very worthwhile.

\section{Acknowledgements}

The first two authors would like to acknowledge the financial support from Research Foundation of the State of S ão Paulo (FAPESP-Brazil). Prof. Brennan acknowledges the support from FAPESP (process number 04/133242-3 Visiting Professor) and the Leverhulme Trust, UK.

\section{References}

[1] Y.J. Yan and L.H. Yam, A synthetic analysis on design of optimum control for an optimized intelligent structure, Journal of Sound and Vibration 249 (2002), 775-784.

[2] D.K. Anthony and S.J. Elliott, On reducing vibration transmission in a two-dimensional cantilever truss structure using geometric optimization and active vibration control techniques, Journal of the Acoustical Society of America 110 (2005), 1191-1194.

[3] W. Gao, J.J. Chen, H.B. Ma and X.S. Ma, Optimal placement of active bars in active vibration control for piezoelectric intelligent truss structures with random parameters, Journal of Computers and Structures 81 (2003), 53-60.

[4] F. Liu and L. Zhang, Modal-Space control of flexible intelligent truss structures via modal filters, Proc. $18^{\text {th }}$ International Modal Analysis Conference (IMAC) 1 (2000), 187-193.

[5] R. Lammering, J. Jia and C.A. Rogers, Optimal placement of piezoelectric actuators in adaptive truss structures, Journal of Sound and Vibration 171 (1994), 67-85.

[6] A. Preumont, J.P. Dufour and C. Malekian, Active damping by a local force feedback with piezoelectric actuators, Journal of Guidance, Control and Dynamics 15 (1992), 390-395.

[7] R. Carvalhal, S. Silva and V. Lopes, Jr., Robust Control Application for Smart Truss Structure, Proc. 23rd International Modal Analysis Conference (IMAC), in CD-ROM, ISBN 0912053895, 2005.

[8] J.Q. Fang, Q.S. Li and A.P. Jeary, Modified independent modal space control of m.d.o.f. system, Journal of Sound and Vibration 261 (2003), 421-441.

[9] L. Meirovitch and H. Baruh, Optimal control of damped flexible gyroscopic systems, Journal of Guidance and Control 4 (1981), $157-163$.

[10] S.P. Singh, H.S. Pruthi and V.P. Agarwal, Efficient modal control strategies for active control of vibrations, Journal of Sound and Vibration 262 (2003), 563-575.

[11] R. Carvalhal, V. Lopes, Jr. and M.J. Brennan, A Comparison of Two Modal Control Strategies for the Active Vibration Control of a Truss Structure, (Vol. 1), Proc. $18^{\text {th }}$ International Congress of Mechanical Engineering (COBEM), 2005, 304-310.

[12] W. Gawronski, Dynamics and Control of Structures: A Modal Approach, Springer Verlag, New York, 1998.

[13] J. Jia, Optimization of Piezoelectric Actuator Systems for Vibration Control of Flexible Structures, Ph.D. Dissertation, Virginia Polytechnic Institute and State University, 1990.

[14] L. Meirovitch, Dynamics and Control of Structures, John Wiley \& Sons, New York, 1990.

[15] B. Xu and J.S. Jiang, Integrated optimization of structure and control for piezoelectric intelligent trusses with uncertain placement of actuators and sensors, Computational Mechanics 33 (2004), 406-412. 

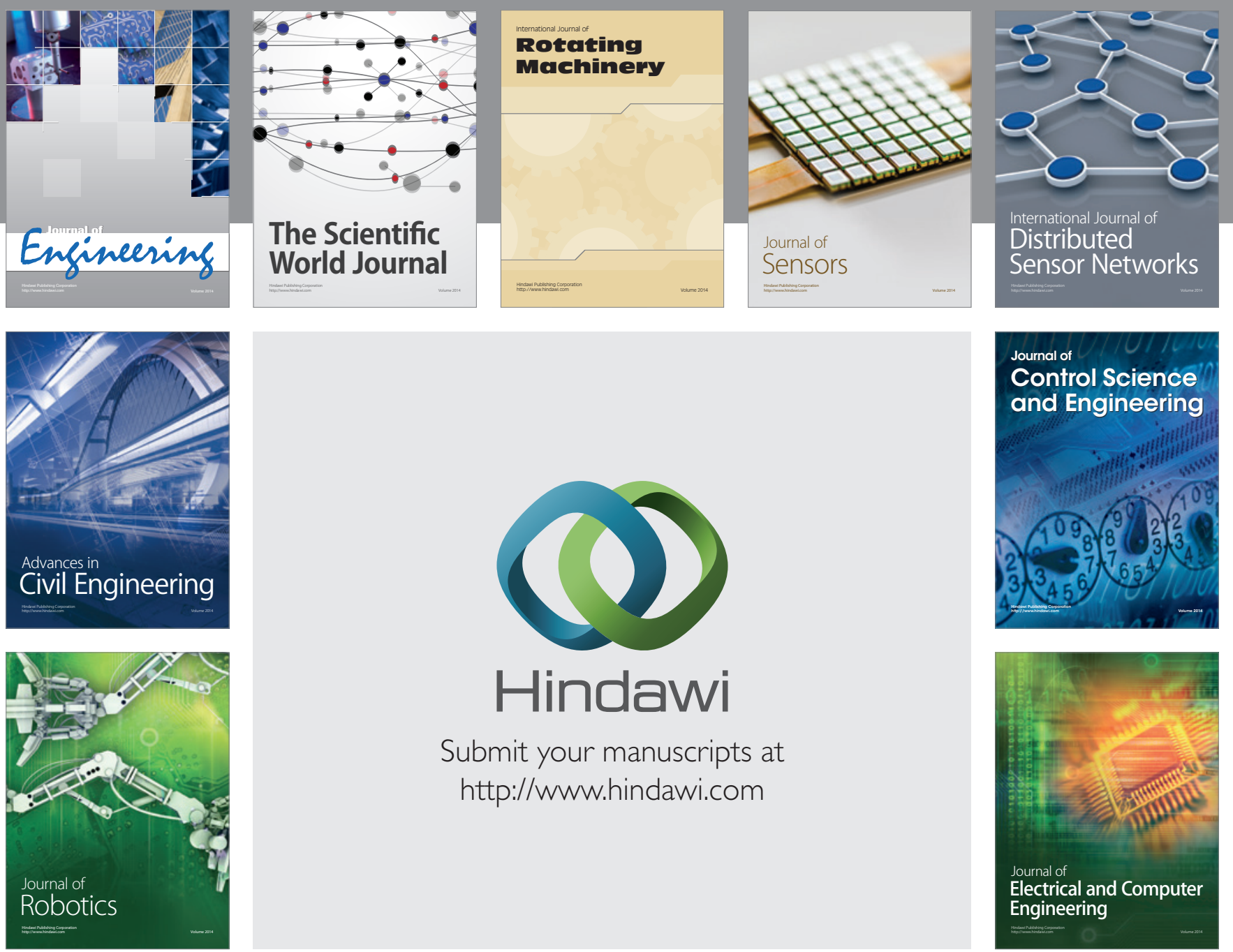

Submit your manuscripts at

http://www.hindawi.com
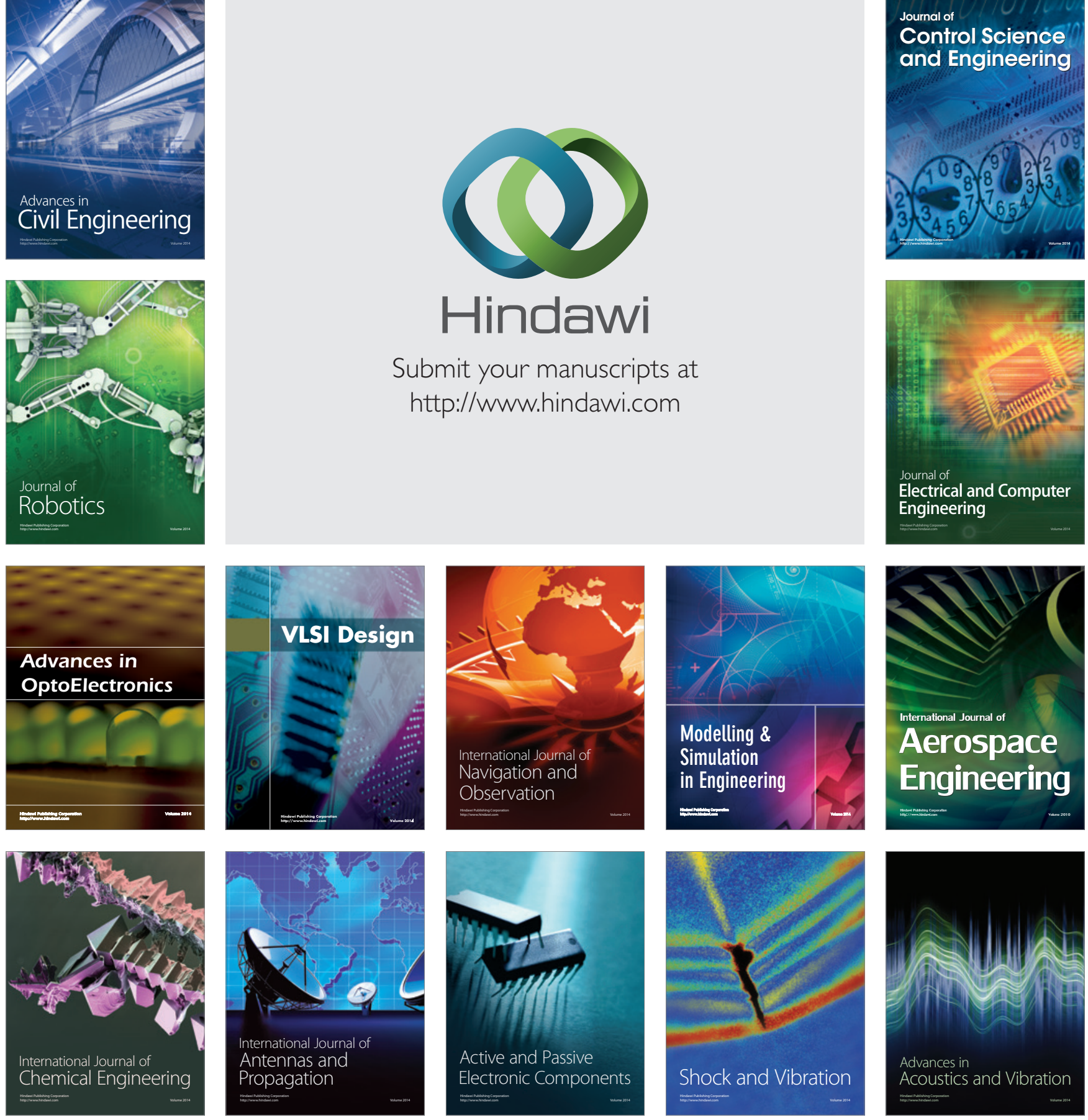